\title{
Les figures paradoxales de l'auteur dans Astérix
}

\section{Nicolas Rouvière}

\section{OpenEdition}

\section{Journals}

Édition électronique

URL : http://journals.openedition.org/recherchestravaux/1344

DOI : 10.4000/recherchestravaux.1344

ISSN : 1969-6434

\section{Éditeur}

UGA Éditions/Université Grenoble Alpes

\section{Édition imprimée}

Date de publication : 15 mai 2004

Pagination : 213-228

ISBN : 2-9518254-3-9

ISSN : 0151-1874

\section{Référence électronique}

Nicolas Rouvière, "Les figures paradoxales de l'auteur dans Astérix », Recherches \& Travaux [En ligne], 64 | 2004, mis en ligne le 20 mai 2019, consulté le 08 septembre 2020. URL : http://

journals.openedition.org/recherchestravaux/1344; DOI : https://doi.org/10.4000/recherchestravaux. 1344 
Nicolas ROUVIÈRE

Université Stendhal-Grenoble 3

\section{Les figures paradoxales de l'auteur dans Astérix ${ }^{\mathrm{I}}$}

Au milieu des années 5o, la bande dessinée franco-belge est confrontée à la question de la naissance juridique et sociale de l'auteur. Le scénariste René Goscinny est sans doute celui qui a œuvré de la manière la plus décisive pour la reconnaissance auctoriale. En 1956, il est à l'origine de la première fronde contre les éditeurs, qui étaient propriétaires de fait des séries et des personnages, et dissuadaient les créateurs de signer leurs œuvres. Son action lui vaut d'être licencié de la World Press, avant d'être rejoint par ses deux camarades, Uderzo et Charlier ${ }^{2}$. Devenu en 1963 co-rédacteur en chef du journal Pilote, après avoir longtemps figuré sur la liste noire des éditeurs, il est le premier à mener une véritable politique d'auteur, en donnant leur chance aux jeunes talents. Qu'il s'agisse de Bretécher, Gotlib, Cabu ou Druillet, tous ont été unanimes pour lui rendre hommage. Dans Astérix, qui est son œuvre majeure, René Goscinny aborde la question de la légitimité auctoriale à travers deux personnages que tout oppose: l'empereur romain et le barde gaulois Assurancetourix. César apparait d'emblée comme une figure de l'auteur, par ses célèbres Commentaires sur la guerre des Gaules, un ouvrage auquel il fait maintes fois référence et qui est parfaitement connu des Gaulois. Il prononce également des phrases historiques qui sont largement reprises par les sujets de l'Empire, ce qui donne lieu à de nombreuses variantes comiques. De fait, à l'image du greffier du Sénat, tout l'appareil d'État est mobilisé pour consigner et diffuser ses citations. César se glisse dans une scénographie auctoriale qui se confond avec celle du pouvoir et se dote d'une persona qui

I. A la demande des éditions Albert René pour l'obtention du copyright: «l'ensemble des propos contenus dans cet article n'engage que son auteur".

2. Voir Marie-Ange Guillaume et José-Louis Bocquet, Goscinny - biographie, Paris, Actes Sud, 1997, p. 86-89. 
s'impose à l'imaginaire social, de telle sorte que se crée autour de lui une mythologie du grand homme. À l'opposé, le barde du village gaulois, qui est auteur, compositeur et interprète de ses propres chansons, échoue immanquablement dans l'affirmation de son talent. Il est cette contre-figure comique de l'auteur narcissique, inféodé à une collectivité qui lui impose rituellement de se taire. Assurancetourix traite de barbares les habitants du village qui dénigrent ses compositions, alors que ses ceuvres sont des parodies de chansons folkloriques ou de succès contemporains. Malgré le sort bien connu qu'on lui réserve dès qu'il ouvre la bouche, le barde a toujours une très haute idée de sa personne. Il adopte la posture de l'artiste incompris, comme si ses créations étaient trop en avance sur leur temps. Entre César et Assurancetourix, entre la mythification et le déni d'existence, à quelle place peut donc légitimement prétendre l'auteur? Voilà une question qui concerne au premier chef les créateurs de bande dessinée eux-mêmes, dans une période où ils affirment leurs prétentions et leurs droits. De fait c'est dans la quête d'une identité auctoriale non usurpée que nous entraîne Astérix, à la recherche d'une place sociale de discours qui désamorce le piège narcissique.

Goscinny et Uderzo s'amusent ainsi à s'auto-représenter dans divers rôles à contre-emploi: dans Astérix aux Jeux Olympiques, les deux auteurs sont caricaturés en magistrats grecs sculptés sur un monument. Vêtus d'une toge, ils touchent les cornes d'un taureau à terre. Leurs noms sont transcrits en alphabet grec, et les mots despotès et tyrannos sont gravés à hauteur de leur bouche.

De l'aveu même d'Albert Uderzo, cette

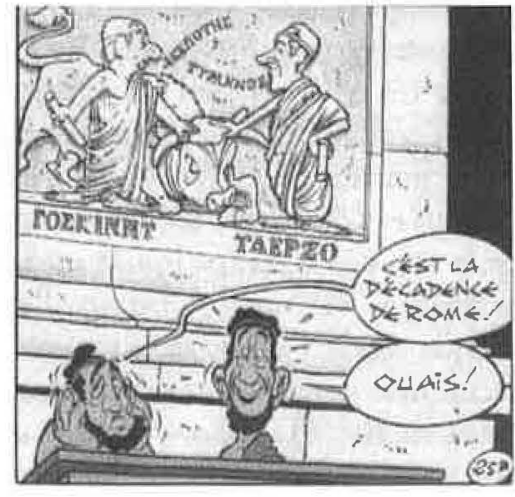

3 représentation est une pure invention. Cela n'exclut pas une référence possible au taureau de Crète vaincu par Héraclès, fondateur mythique des Jeux Olympiques. Robert Graves note que le combat contre un taureau faisait partie du rite de couronnement en Grèce. Le contact de la corne conférait au roi sacré le pouvoir sur les saisons de l'année qu'il gouvernait ${ }^{4}$. A travers cette représentation, Goscinny et Uderzo identifient la place de l'auteur à celle du pouvoir et se posent ironiquement en maîtres légaux du sens, en détenteurs

3. Goscinny et Uderzo, Astérix aux Jeux olympiques, Paris, Hachette, 1999 ( $\mathrm{I}^{\mathrm{r}}$ édition, Dargaud, 1968), p. 29, c. 10. (2003-Les éditions Albert René/Goscinny-Uderzo.

4. Robert Graves, Les Mythes grecs, Paris, Fayard, 1967 , tome 2, I23.I, p. 102. 
absolus des significations. Ils imitent la posture de César, qualifié de « tyran " dans l'album Astérix gladiateur. De fait, l'empereur entend devenir le maître de la vérité de l'histoire. Sa version personnelle de la défaite d'Alésia, qu'il raconte en parlant de lui-même à la troisième personne pour feindre l'objectivité, s'oppose radicalement à celle des auteurs's. De plus il fait tout pour que ses échecs restent secrets, tel le triomphe d'Abraracourcix sur le bouclier de Vercingétorix, ou sa défaite militaire contre les Belges.

Contre cette figure césarienne qui entend rester maîtresse du sens, la quête de l'identité auctoriale s'oriente dans Astérix vers des figures d'interprètes.

Les auteurs se posent ainsi plus modestement en simples scribes traducteurs, transcrivant la parole en images. Dans Astérix et Cléopâtre, le scribe de l'architecte Numérobis, qui parle les langues "vivantes" comme le grec, le latin ou le celte, apparaît sous les traits de René Goscinny habilement caricaturé. Le druide le questionne sur sa situation sociale, allusion comique au statut de scénariste de bande dessinée au début des années 60 .

PANORAMIX: C'est une bonne situation ça, scribe?...

MisENPLIS: Oh c'est une situation assise... accroupie plutôt ${ }^{6}$.

Le calembour sur la position du scribe assis en tailleur laisse entendre que, loin d'être solide et stable, la situation d'auteur de B.D. est plutôt inconfortable, contrairement à celle de scribe dans le royaume d'Égypte. Le druide se montre d'ailleurs curieux d'un tel devenir professionnel:
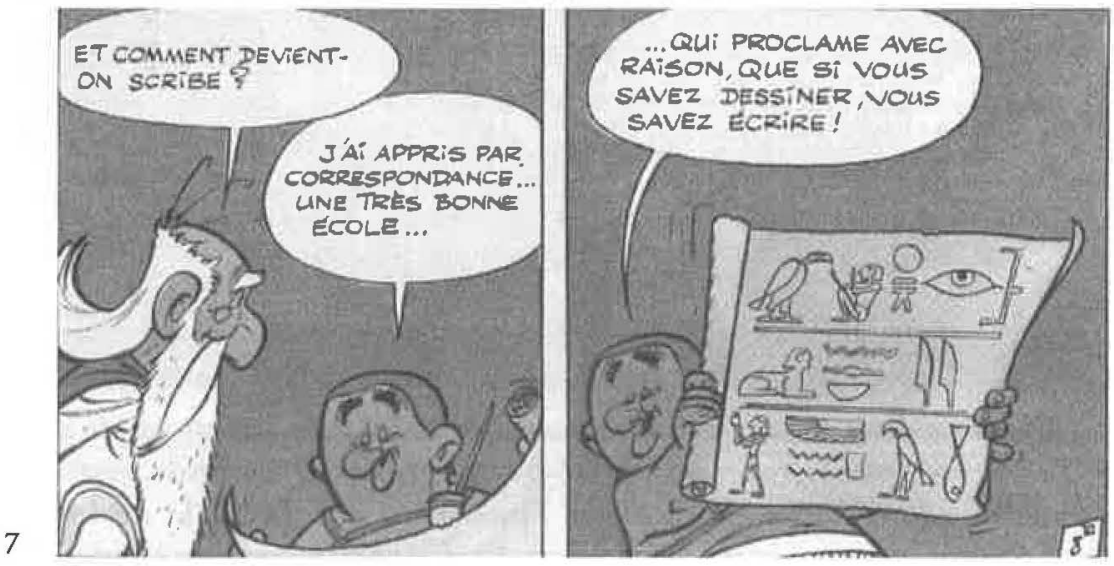

5. Pour comparer les deux versions de la défaite d'Alésia, voir Le Domaine des dieux, p. 5, c. I-2, et Le Bouclier arverne, p. 5, c. I-2.

6. Goscinny et Uderzo, Astérix et Cleopâtre, Paris, Hachette, I999 ( $\mathrm{I}^{\mathrm{r}}$ édition: Dargaud, 1965), p. I2, c. 8.

7. Ibid.p. I2, c. 9-10. (C2003-Les éditions Albert René/Goscinny-Uderzo. 
Le calembour sur le mot " correspondance" qui fait allusion ici aux méthodes d'apprentissage à distance des langues étrangères, pose l'auteur de B.D. en traducteur. Il est celui qui transcrit la langue de l'oral en une langue d'images, et fait correspondre les deux systèmes. Du reste, dans Astérix et Cléopátre, la langue hiéroglyphique des Égyptiens est réinventée sous une forme graphique fantaisiste qui rappelle l'art séquentiel de la bande dessinée. Tantôt le narrateur donne la traduction de ces curieuses itérations d'images, tantôt il avoue ses limites de traducteur. A l'image du scribe Goscinny, c'est alors au lecteur de deviner et de transcrire le contenu des échanges.

Si l'auteur est un traducteur-interprète, la bande dessinée met en scène nombre d'imposteurs. En témoigne la figure de l'augure, mot dont l'étymologie, selon Émile Benveniste, procède tout comme le mot "auteur" du verbe latin augeo ${ }^{8}$. L'augure est celui qui fait surgir le sens des présages et révèle les intentions divines. Le devin qui est de passage dans le village se pose ainsi en interprète autoproclamé des dieux. Il prétend lire l'avenir dans les entrailles des animaux et propose de sacrifier Idéfix, car il " lit» très bien dans les chiens.

Goscinny et Uderzo filent la métaphore de la lecture pour euphémiser la réalité meurtrière du sacrifice et tirent de cette substitution les meilleurs effets comiques.

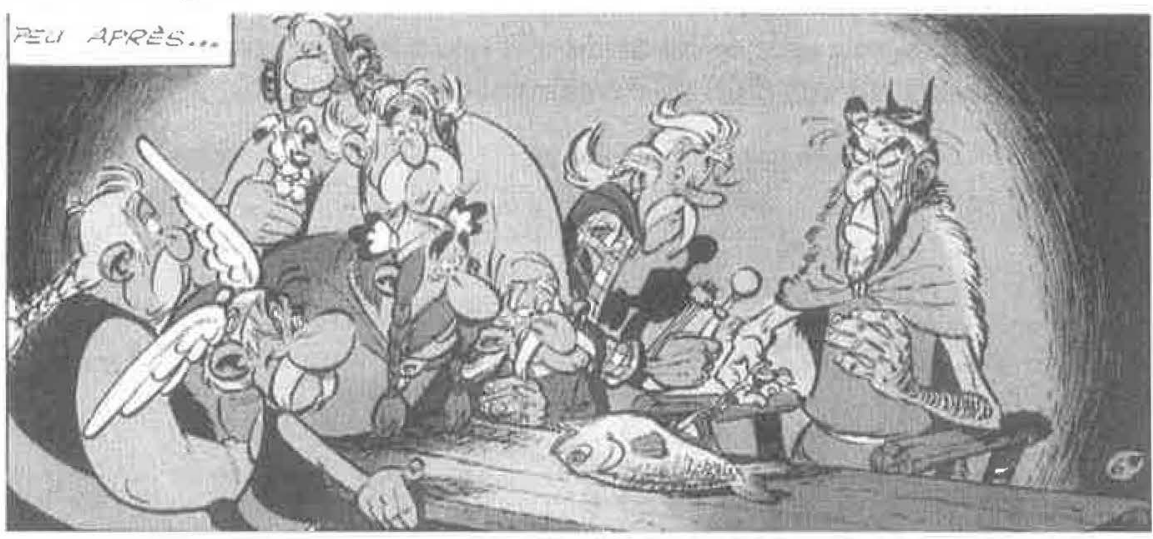

La séance de divination, qui est un pastiche de La leçon d'anatomie du docteur Tulp peint par Rembrandt en 1632, est un rituel morbide visant à impressionner l'assistance. Loin d'être l'interprète des dieux, le devin est l'auteur unique de ses propres prédictions. La divination consiste à satisfaire le désir narcissique du client, en cautionnant l'enlacement subjectif avec

8. Émile Benveniste, Le Vocabulaire des institutions indo-européennes, Paris, Minuit, 1969, II, p. I49-I50.

9. Le Devin, Paris, Hachette, 1999 (Dargaud, 1972), p. 90, c. 10. (C2003-Les éditions Albert René/Goscinny-Uderzo. 
l'image rêvée de soi. Et Obélix se laisse facilement prendre au piège, dès qu'on lui prédit l'amour d'une belle jeune femme blonde.

Si l'auteur ne saurait être un augure, un autre contre-modèle consiste à se faire l'interprète-relais du pouvoir, son porte-parole, le traducteur de ses valeurs. Ainsi Goscinny et Uderzo sont caricaturés en notables romains siégeant aux côtés du Préfet de Condate, au premier rang d'une représentation théâtrale. Le dessinateur rassure ce dernier sur l'intérêt du spectacle, tandis que le scénariste, figuré à droite de la vignette, fait rire ses voisins par quelque bon mot.

IO

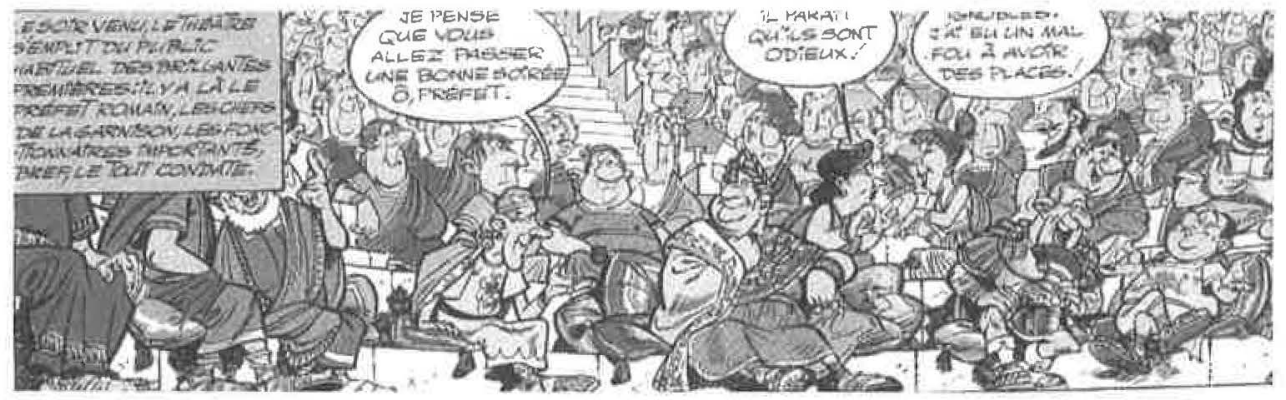

Eléonoradus, l'auteur de la pièce en question, veut révolutionner l'art et la société, à l'image des auteurs contemporains du Living Theatre:

Je veux renouveler l'art dramatique! Nous avons un message! Une mission!

Nous devons choquer le public! Le sortir de sa léthargie! Tout doit être spontané! II

En fait le public assiste avec plaisir à la pseudo-contestation libertaire de ses propres valeurs bourgeoises et le gouverneur, loin d'être choqué par le spectacle, se réjouit de la fausse polémique ainsi provoquée, comme d'un luxe que s'offre la classe au pouvoir. Car la collusion entre la société et les artistes est totale. Le spectateur qui crie au scandale est un acteur de la troupe dissimule dans les gradins, tandis que le discours du metteur en scène, loin d'avoir pour effet d'opposer le public et la scène, suscite une identification complaisante:

ELEONORADUS: Vous êtes laids! Nous sommes tous laids, mais moins que vous! Beuh!

UNE SPECTATRICE : C'est insupportable de vérité!... ${ }^{12}$

Io. Goscinny et Uderzo, Astérix et le chandron, Paris, Hachette, 1999 (Dargaud, 1969), p. 30, c. 9. @2003-Les éditions Albert René/Goscinny-Uderzo.

II. Ibid. p. 29, c. 2.

I2. Ibid. P. 3I, C. 3-4. 
Il suffit d'observer la mine réjouie du Préfet mise en valeur par quatre plans rapprochés successifs, pour se persuader qu'à son insu l'auteur rebelle sert autant le pouvoir en place que les auteurs officiels du premier rang. En offrant à la bonne société le luxe de contester les repères moraux, Eleonoradus conforte le narcissisme social de l'oligarchie cultivée à laquelle il s'adresse.

Un incident cependant vient briser la satisfaction narcissique du public, flatté du miroir qu'on lui tend. Eleonoradus en effet demande à Obélix d'improviser un mot à l'avant- scène, pour que le spectacle se termine sur un cri de spontanéité. Seul face au public et tétanisé par le trac, Obélix, dans un état second, finit par prononcer son leitmotiv bien connu: "Ils sont fous ces Romains!».

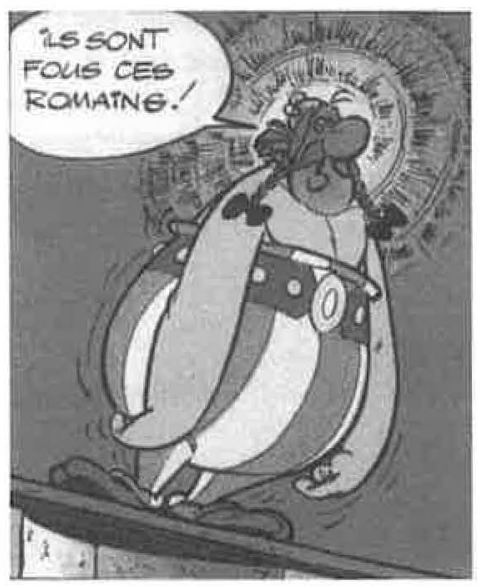

13

La spontanéité de cette parole, prononcée dans un état semi-conscient, ne fait aucun doute. Conformément au vou du Living Theatre, Obélix a puisé dans ses ressources psychiques les plus profondes pour exprimer la vérité qu'il porte en lui, ce qui tranche radicalement avec les fausses improvisations qui précèdent. Le prédicat de folie s'avère d'autant plus subversif que l'intrusion du pronom cataphorique "ils", relayé par le déictique "ces", désigne les Romains sur le mode de l'objet et les rejette dans une altérité radicale. Brusquement s'écroule pour cette bonne société la connivence qui consiste à se donner le spectacle de sa propre contestation, sur le mode convenu du «nous» et du "vous». Aussitôt le Préfet crie au crime de lèse-majesté et fait donner la troupe :

COMMENT , C'EST INADMISSIBLE EMBARQUEZ-MOI CES IMBÉCILES QUI OSENT SE MOQUER DE L'AUTORITÉ ! ${ }^{14}$

En écho au vou d'Antonin Artaud de faire retour sur scène vers une parole primitive qui soit une force mythopoïétique ${ }^{15}$, les auteurs rejouent ici sous une forme caricaturale l'entrée première dans le langage. La parole d'Obélix rappelle la parole de l'enfant qui dans un acte inaugural crée le vis-à-vis de l'apparition et rend soudain possible que le monde apparaisse comme un

13. Ibid. p. 32, c. 3. (C2003-Les éditions Albert René/Goscinny-Uderzo.

14. Ibid. p. 32, c. 5 .

15. Voir Monique Borie, Antonin Artaud: le théatre et le retour aux sources, Paris, Gallimard, 1989, p. 26-30. 
objet projeté devant soi. Or, c'est une position souveraine que de faire corps avec le pouvoir de séparer, désigner et classer. Poser pour la première fois quelque chose d'extérieur en le désignant, c'est pour l'enfant œuvrer dans l'insu ex auctoritate, depuis la place qui présente, exhibe, donne le signe. Obélix est en position d'enfant-auteur qui fait surgir le monde, l'objective en s'en séparant, et construit dans la représentation la figure de l'autre. Cette entrée dans la parole, rejouée ici de façon comique sous une forme quasi inaugurale, postule une position proprement intenable pour le pouvoir en place, qui ne supporte pas d'être présenté soudain à soi depuis cette place souveraine.

Voilà qui nous fait quitter le monde des interprètes patentés pour celui de l'enfance de l'art, où l'auteur est un enfant expérimentateur, ignorant le sens de ce qu'il fait.

Ainsi, dans l'album Le Combat des chefs, les druides Panoramix et Amnésix, qui ont tous deux perdu la mémoire et la raison, depuis qu'ils ont reçu un menhir sur la tête, préparent des potions fantaisistes afin d'obtenir sur eux-mêmes les coloris cutanés les plus farfelus:

PANORAMIX: Si nous mettions tous les ingrédients qui nous restent dans une seule marmite? ça serait rigolo monsieur. Je parie que nous serons à carreaux rouges et verts!

AMNESIX: Ou à pois bleus sur fond jaune! HiHIHIHIHI!

AMNESIX: Oh, la belle bleue!

PANORAMIX: Oh, la belle rouge! ${ }^{16}$

Tout comme Obélix précédemment, les druides se découvrent comme pouvoir de séparer, et font surgir l'extériorité sur le mode de l'objet. Ils inaugurent la place souveraine de l'enfant qui joue à déléguer son corps dans l'image et pour la première fois le désigne comme un théâtre de signes. Il s'agit d'un passage autopoḯtique par lequel les personnages entrent dans la séparation d'avec eux-mêmes en s'inventant une corporalité seconde. Ils expérimentent dans l'insu le pouvoir auctorial de l'enfant faisant surgir la manifestation de la présence à soi.

Obélix apparaît, quant à lui, comme un curieux artisan fabriquant des menhirs sans utilité sociale ni valeur cultuelle, et semble agir machinalement sans développer la moindre réflexion sur le sens de son activité. Cependant cette forme d'" art brut » est parfaitement intégrée à l'économie du village et peut servir de monnaie d'échange. Alors qu'il est indissociable de la nature infantile du personnage, le menhir devient paradoxalement un emblème de

16. Le Combat des chefs, Paris, Hachette, 1999 (Dargaud, 1966), p. 4I, c. 4-5 et p. 35, c. ro. 
l'identité collective. Ainsi le chef s'oppose officiellement à ce que le menhir prenne la forme de l'obélisque égyptien. Curieux destin collectif pour cet objet mal dégrossi qui rappelle par sa forme phallique la nature pulsionnelle du personnage.

Ces figures paradoxales d'auteurs-enfants œuvrent dans l'insu, au point que leur action prend l'allure d'un acte manqué ou d'une gaffe. Ainsi, dans l'album Astérix et Cléopatre, Obélix, en escaladant le Sphinx de Gizeh, décroche accidentellement le nez de la statue, réalisant sans le savoir ce dont tout un peuple était en attente: un monument qui représente de nouveaux fondements pour le politique. En effet, la célèbre statue du pharaon Kephren symbolise la communion totale entre le roi et le dieu solaire ${ }^{17}$. Nous avons affaire ici à la figure sacrée d'un Père mythique qui est le pharaon érigé en dieu totémique. Or l'action des Gaulois équivaut à un changement de totem, au remplacement d'un fondement théologico-politique par un autre fondement. Le décrochage du nez stipule qu'aucune figure humaine, désormais, ne peut se confondre avec le principe absolu. Les Gaulois ont le souci que le nez ne soit jamais retrouvé, l'enfouissent sous le sable, puis partent précipitamment comme des criminels. Cet épisode rappelle la question du meurtre du Père évoquée dans Totem et tabou. On retrouve la culpabilité des fils analysée par Freud, ainsi que la tentative rétrospective d'étouffer ce sentiment, une fois le crime accompli, en renonçant à cueillir le fruit de cet acte, la toutepuissance symbolique du nez. Or, un personnage prétend à cette place absolue, il s'agit de Cléopâtre. Car la scénographie du pouvoir identifie la reine à une divinité. Quand elle rend visite aux bâtisseurs, elle apparât au sommet d'un char monumental représentant un gigantesque sphinx d'or qui renvoie
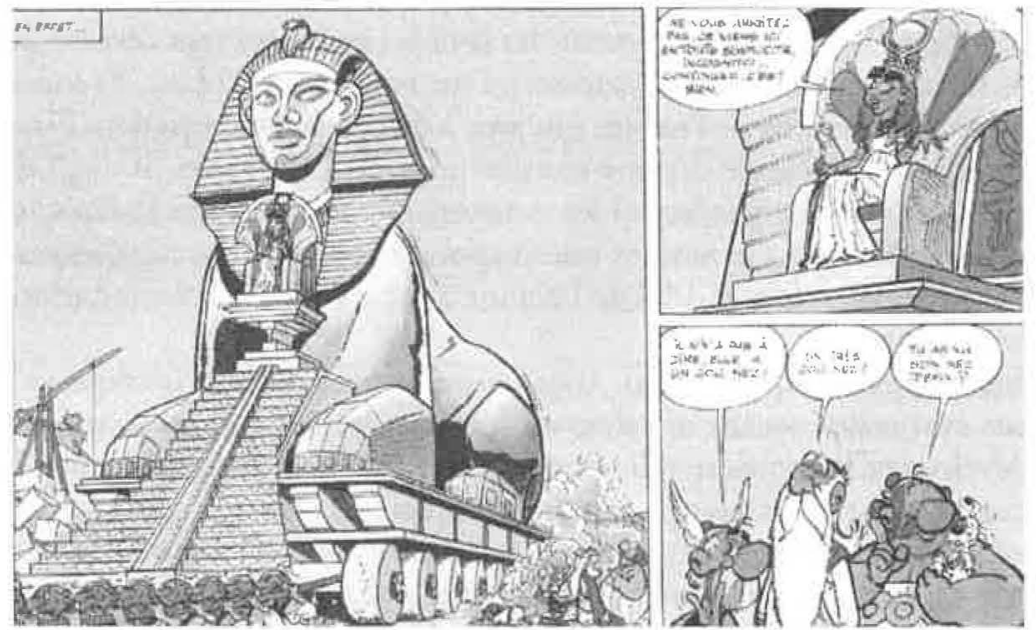

17. Voir Claire Lalouette, Au Royaume d'Egypte : le temps des rois dieux, Paris, Flammarion, I99I, p. I22.

18. Goscinny et Uderzo, Astérix et Cléopattre, Paris, Hachette, 1999 (Dargaud, 1965), p. 27, c. 3-5. (C) 2003 - Les Éditions Albert René / Goscinny-Uderzo. 
directement à la représentation du Sphinx de Gizeh. En reprenant cette symbolique, Cléopâtre tend à s'instituer au regard de ses sujets comme une divinité incarnée. Du reste, ce qui trône au sommet du char, c'est véritablement son nez, clairement mis en valeur par les commentaires des Gaulois.

Cléopâtre porte le nez symbole de toute-puissance qui manque au Sphinx de Gizeh. Or, le pouvoir théocratique de la reine a fait son temps. César constate que le peuple égyptien est décadent, et ne construit plus rien. Offusquée, Cléopâtre a beau ordonner la construction d'un palais pour César, l'architecte Numérobis s'avère incapable de le construire "moderne", c'est-à-dire sous une forme autre que pyramidale.

C'est donc Obélix qui, le premier, et à son insu, érige la modernité politique dans la représentation. En décrochant le nez de la statue, il donne un nouveau visage au fondement du pouvoir.

Aussitôt, l'ensemble des marchands qui constatent la disparition du nez monumental se hâtent de casser celui de leurs statuettes en série.

La nouvelle figure du fondement politique prend ainsi statut sur la scène sociale grâce au relais de l'art et du commerce. Obélix, l'enfant gaffeur, a œuvré sans le savoir comme un véritable auteur législateur.

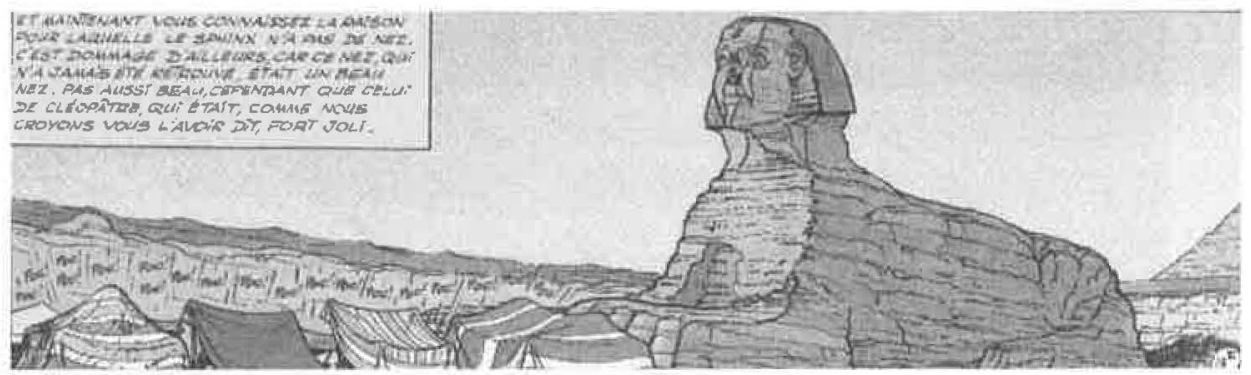

19

A travers ces figures d'enfants auteurs, non seulement la part de l'insu dans la création s'avère donc déterminante, mais encore la portée collective de ce qui est créé invalide toute prétention à la maîtrise totale du sens. Voilà qui permet sans doute d'opposer deux modèles de bande dessinée.

Le premier est à l'image de l'enceinte érigée par les Gaulois pour défendre le palais construit par Numérobis. Elle se compose de blocs de pierre espacés, qui rappellent très exactement les vignettes d'un strip de B.D., avec ses vides inter-iconiques:

19. Ibid. p. 22, c. 10. ( 2003 - Les Éditions Albert René / Goscinny-Uderzo. 


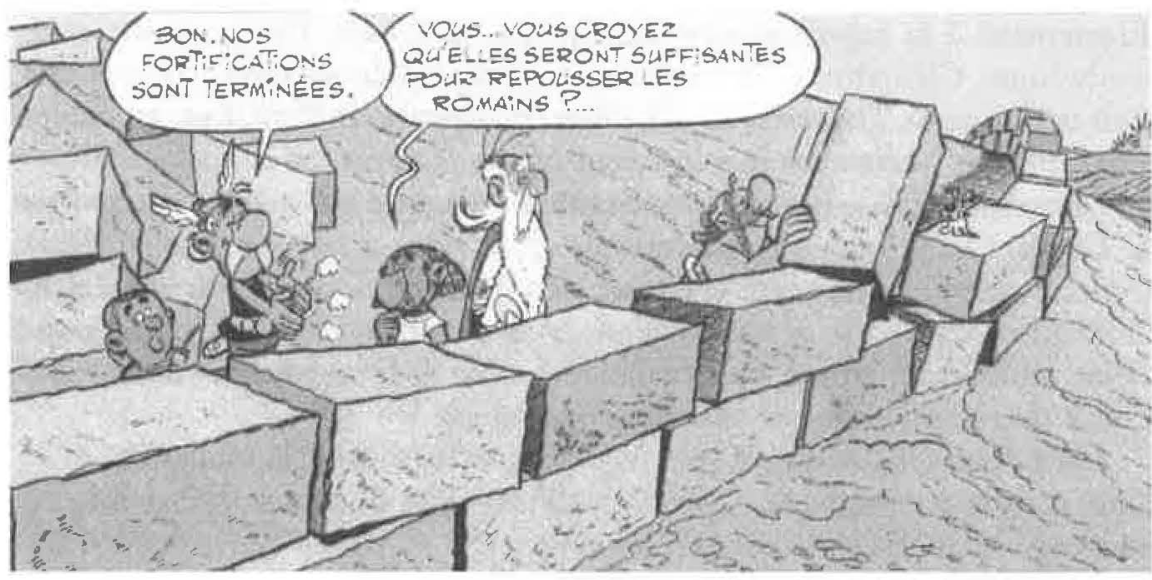

20

Le second modèle est à l'image de la tortue romaine qui attaque le bâtiment. Elle évoque la géométrie d'une planche de bande dessinée, mais sous la forme agressive d'un blindage de boucliers rectangulaires hermétiquement joints:

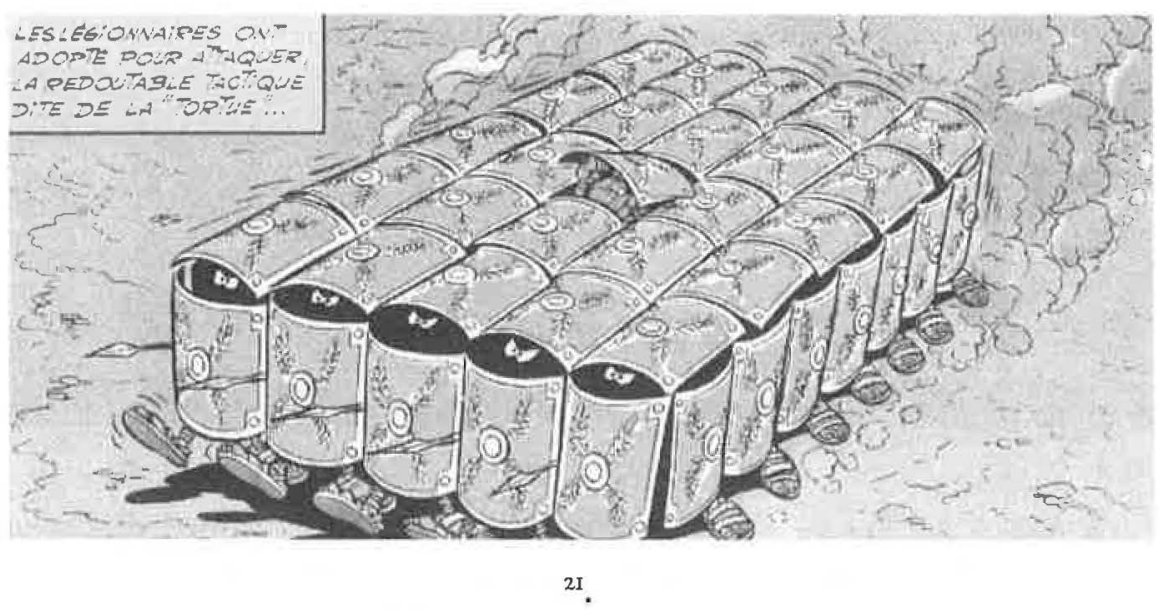

Il y a là une ambition auctoriale qui s'échafaude: créer une B.D. qui ménage du jeu, contre toute prétention à fermer absolument le sens. Mais cette enceinte n'est pas seulement une réserve de vignettes qui font mouche par le comique, elle est là pour défendre une architecture qui traduit un nouveau rapport collectif au pouvoir, affranchi de la théocratie pyramidale.

20. Ibid. p. 39, c. 2.(C) 2003 - Les Éditions Albert René / Goscinny-Uderzo.

2I. Ibid. p. 39, c. 4.@ 2003 - Les Éditions Albert René / Goscinny-Uderzo. 


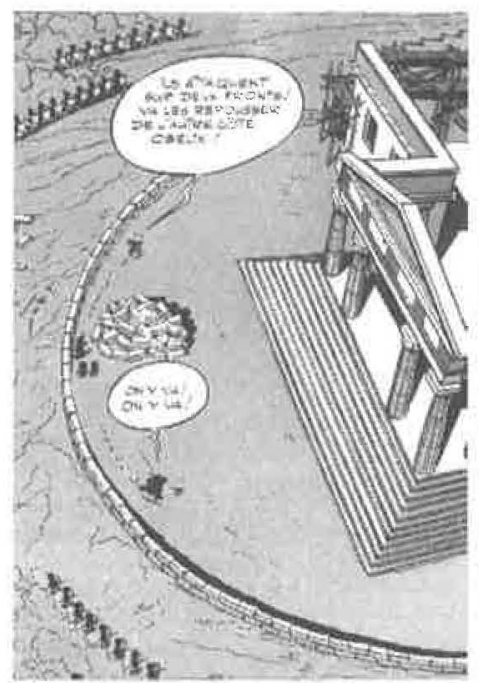

22

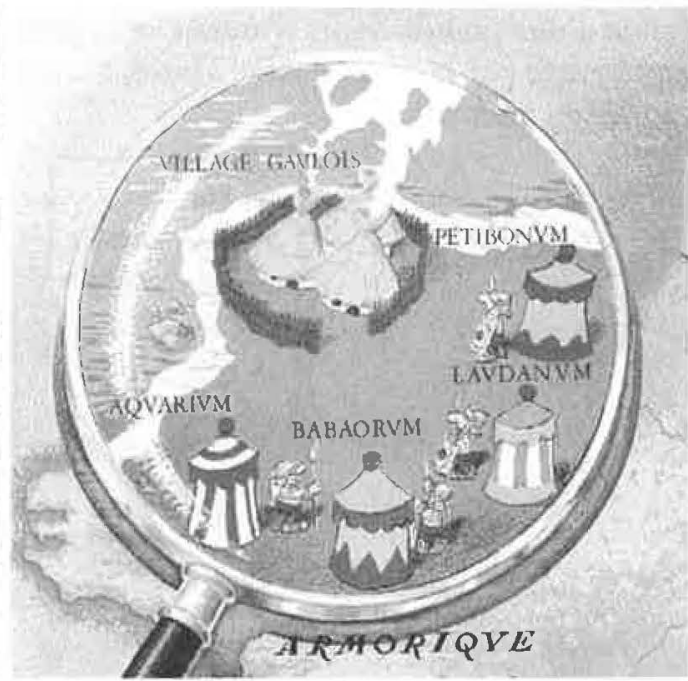

23

Or, l'enceinte circulaire rappelle très exactement celle du village gaulois cerné par les camps romains. Selon cette logique métatextuelle, tout se passe comme si la bande dessinée, au-delà de sa visée comique, proposait un second pacte de lecture: modéliser des constructions institutionnelles, comme celles du village gaulois ou du royaume d'Égypte; cerner ce qui fait leur viabilité, comprendre ce qui les fait tenir, en les confrontant à d'autres modèles concurrents, tel le système romain.

Cependant des créateurs de B.D. comme Goscinny et Uderzo peuvent-ils mettre en forme dans la fiction une étude comparative des sociétés et de leurs fondements, sans prétendre rivaliser avec les grands auteurs de la littérature? C'est poser à nouveau la question de la légitimité des places-de discours, par rapport à un genre paralittéraire jugé mineur.

L'album Astérix chez les Belges aborde le problème. Une célèbre citation de César extraite de La Guerre des Gaules provoque la colère du chef Abraracourcix: "de tous les peuples de la Gaule, ce sont les Belges les plus braves ". Outré, le chef du village décide de mener aussitôt une expédition en Belgique pour faire la preuve de la bravoure gauloise. Il s'agit de se comparer à ce peuple voisin pour comprendre ce qui fait sa prétendue supériorité. Or, la rivalité entre Belges et Gaulois semble être la métaphore d'une rivalité entres les écoles belge et française de la bande dessinée, pour se mesurer à

22. Ibid. p. 40, c. I. @ 2003 - Les Éditions Albert René/ Goscinny-Uderzo.

23. Goscinny et Uderzo, Astérix le Gaulois, Paris, Hachette, 1999, p. 3. (C) 2003 - Les Éditions Albert René / Goscinny-Uderzo. 
l'aune d'une grande figure d'auteur en la personne de César. L'école belge est représentée par la célèbre série Tintin, à travers les Dupont et Dupond caricaturés en guerriers:

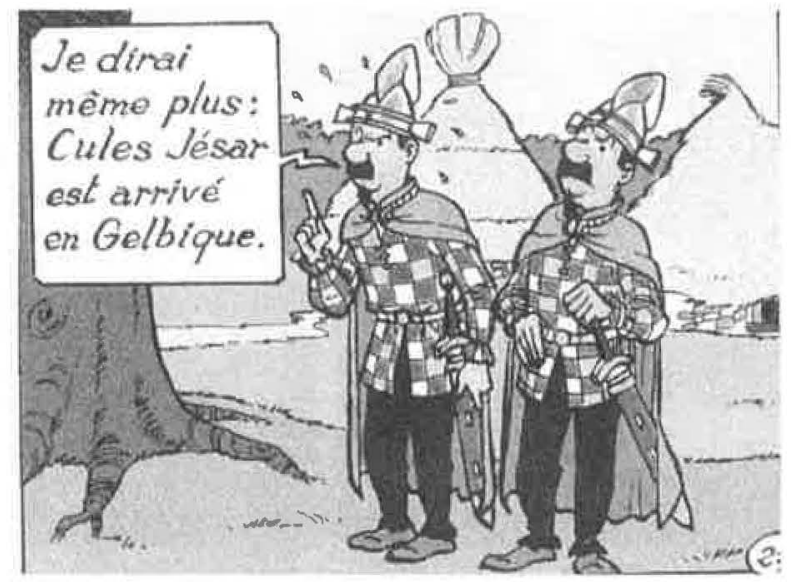

24

D'emblée le druide juge l'idée de cette confrontation absurde, tandis que le barde plaide pour la qualité de la création:

PANORAMIX: Si les Belges sont braves, tant mieux pour eux et tant pis pour César. Occupons-nous de nos affaires!

ASSURANCETOURIX: Notre druide a raison; les qualités artistiques sont plus importantes que la force brutale ${ }^{25}$.

Occupons-nous en somme de notre ouvrage en veillant à sa qualité artistique, sans jalouser les lauriers que l'on décerne à la série belge. Mais Abraracourcix veut que son nom reste dans l'histoire et quitte la table du conseil en faisant valoir son départ solitaire comme le signe électif de sa propre grandeur. Il cède au narcissisme auctorial de l'auto-représentation. Aussi lorsqu'il fait son baluchon entame-t-il avec complaisance le récit de sa propre épopée:

Oui mimine, je pars et je pars seul... Je dois défendre l'honneur du village et l'honneur de l'ancien combattant de Gergovie que je suis... je sais que c'est dur, mais quels que soient les dangers qui... ${ }^{26}$

24. Goscinny et Uderzo, Astérix chez les Belges, Paris, Hachette, I999 (Dargaud, 1979), p. 3I, c. 8. (c) 2003 - Les Éditions Albert René / Goscinny-Uderzo.

25. Ibid. p. I0, c. 5 .

26. Ibid. p. II, c. 2-3. 
La réponse de Bonemine a tôt fait de soumettre cette emphase à l'épreuve du burlesque:

En rentrant, passe chez Ordralfabétix. Tu prendras des maquereaux, mais pas trop rances, cette fois-ci ${ }^{27}$.

Voilà à quoi s'expose quiconque veut créer un mythe de sa propre personne. On ne joue pas impunément à César. Car César est bien cette figure de l'auteur majuscule que chacun prend pour référence ou veut imiter, au point que Belges et Gaulois le choisissent comme arbitre.

De fait, en tant que double figure de l'auteur et du pouvoir, le célèbre personnage se scinde en deux figures séparées, celles de l'écrivain Victor Hugo et du conquérant Napoléon. Ainsi le récit de la bataille qui oppose les Belges aux légions romaines est-elle une vaste parodie burlesque du récit de la défaite de Waterloo dans L'Expiation.

Le soir tombait; la lutte était ardente et noire.

César avait l'offensive et presque la victoire;

Il tenair les Belges acculés sur un bois.

[...]

L'espoir changea de camp, le combat changea d'âme.

$[\ldots]$

Et triarii, principes aux caligae de cuir,

Hastati dont Rome faisait des légionnaires,

Vélites sagitarii qui traînaient leur crinière,

Portant des clipeus et jambières de métal,

Tous ceux d'Alésia et ceux de Pharsale...

[...]

Comprenant qu'ils allaient drôlement déguster,

Leur bouche d'un seul cri, dit:

C'EST PAS UN PEU FINI! ARRETEZ ! ${ }^{28}$

La référence à Victor Hugo est emblématique d'une conception de l'auteur qui s'attache à faire coïncider la personne et le personnage public d'écrivain. Alain Vaillant rappelle ce rêve unitaire de Hugo:

Édifiant son ouvre comme d'autres leur personnage public, reversant dans l'écrit l'éphéméride de sa vie et conformant son action personnelle aux exigences éthiques et esthétiques de son écriture, Victor Hugo a assigné au livre publié le seul rôle qui lui permît de concilier le réel et le signe, être l'homme:

Tout homme qui écrit écrit un livre; et ce livre, c'est lui ${ }^{29}$.

27. Ibid. p. II, c. 3 .

28. Ibid. p. 43-45. A comparer avec le texte original de "L'Expiation" (II), dans Les Chattiments $(\mathrm{V}, \mathrm{13})$.

29. Alain Vaillant, "Entre personne et personnage: le dilemme de l'auteur moderne", Colloque de Cerisy-la-Salle (4-8 octobre 1995): "L'Auteur", actes publiés sous la direction de Gabrielle Chamarat et Alain Goulet, Presses universitaires de Caen, 1996, p. 48-49. La citation de Hugo reprise par Alain Vaillant est extraite de la Préface à l'édition ne varietur, I880. 
La parodie burlesque de L'Expiation déconstruit précisément cette posture auctoriale, aussi mystificatrice que celle de César. Assez des mythes personnels d'écrivains. Telle est la leçon qui ressort lorsque Abraracourcix et le chef belge Gueuselambix barrent la route à l'empereur dans sa retraite et lui demandent de les départager. César, pris de colère, leur crie son incapacité à trancher dans leur querelle de fous. Les deux hommes se reconnaissent soudain comme deux comiques ridicules et sont gagnés par un irrésistible fou rire. Le pastiche final du tableau de Bruegel ${ }^{30}$ vient alors consacrer l'acceptation joyeuse et bon enfant d'une même éthique auctoriale partagée, celle du comique et de la culture populaire de l'image, contre les mythes d'auteur de la grande littérature.

Reste que face à la tentation césarienne, un garant ne serait de trop, pour désamorcer le piège narcissique. C'est peut-être alors vers le personnage du barde Assurancetourix qu'il faut se tourner, cette figure de l'auteur censuré.

Assurancetourix n'est pas un bouc émissaire. La violence qu'il subit de la part des villageois ne correspond pas aux stéréotypes de la persécution définis par René Girard ${ }^{39}$. Le barde n'est pas assommé ni bâillonné dans un contexte de crise communautaire, mais au contraire au cours du grand banquet final qui célèbre l'unité retrouvée du village. Personne ne cherche non plus à le martyriser sous prétexte qu'il est efféminé ou mène une vie à part au sommet de sa hutte. Assurancetourix se voit confier au contraire des fonctions institutionnelles importantes, puisqu'il joue le rôle d'instituteur et arbitre même le débat politique lors de l'élection du chef. S'il est frappé ou bâillonné, c'est uniquement parce que son chant est insupportable.

Sa voix fait fuir universellement tous les êtres vivants, y compris les bêtes sauvages de la forêt et les lions du cirque. Il s'agit là d'une arme absolue équivalente à la potion magique, puisqu'elle libère les villageois des envahisseurs normands ou des colons romains qui s'installent à proximité dans $L e$ Domaine des dieux. Cette voix est qualifiée par le narrateur d'«innommablen:

Assurancetourix, c'est le barde. Les opinions sur son talent sont partagées: lui, il pense qu'il est génial, tous les autres pensent qu'il est innommable ${ }^{32}$.

La voix du barde ouvre sur quelque chose qu'il n'est pas possible de nommer, elle notifie une véritable impasse de la représentation. Ainsi le chef normand n'a pas de mot pour désigner ce qu'il entend, et ne peut qu'évoquer l'effet produit sur son corps:

30. Bruegel l'ancien, Le Repas de noces (1568), ibid. p. 47.

31. René Girard, Le Bouc émissaire, Paris, Grasset, 1985, p. 30-75.

32. Voir "Quelques Gaulois", en préambule de chaque album p. 4. 
À l'idée d'entendre ça encore une fois, mes genoux tremblent, mes dents claquent, des sueurs froides couvrent mon front et mon estomac se noue! 33

Les mots sont toujours en deçà de l'horreur inouie de ce chant, comme en témoignent les pleurs du geôlier romain dans Astérix gladiateur. En prière à genoux et les mains jointes, le légionnaire s'adresse au barde comme à une instance qui le dépasse. Car Assurancetourix fait entrevoir qu'il existe une dimension autre, la dimension de ce qui n'a pas de nom. Et il faut redoubler la négativité, c'est-à-dire faire en sorte que l'innommable se taise, pour que la parole joyeuse du banquet soit possible. Bâ̂llonné, le barde rappelle que quelque chose se tait absolument derrière le langage et à travers lui. La parole n'existe peut-être même qu'au nom de ce vide qui la fonde, cet innommable qu'elle recouvre.

Loin que la communication soit un rapport duel entre émetteur et récepteur, il existerait une instance tierce de la communication, représentée ici par le barde, qui emblématise ce qui se tait absolument derrière les mots. Ligoté auprès de son arbre, Assurancetourix a ainsi valeur de totem pour le village. Il crée la mise en scène du vide, constitutif de la parole. Sous son égide, la communication peut battre son plein. Le rituel du bâillon infligé à Assurancetourix fait surgir l'espace tiers de l'écart, la place qui tient l'être de parole indéfiniment à distance de soi et en retrait du monde, faisant ainsi obstacle à la scène narcissique.

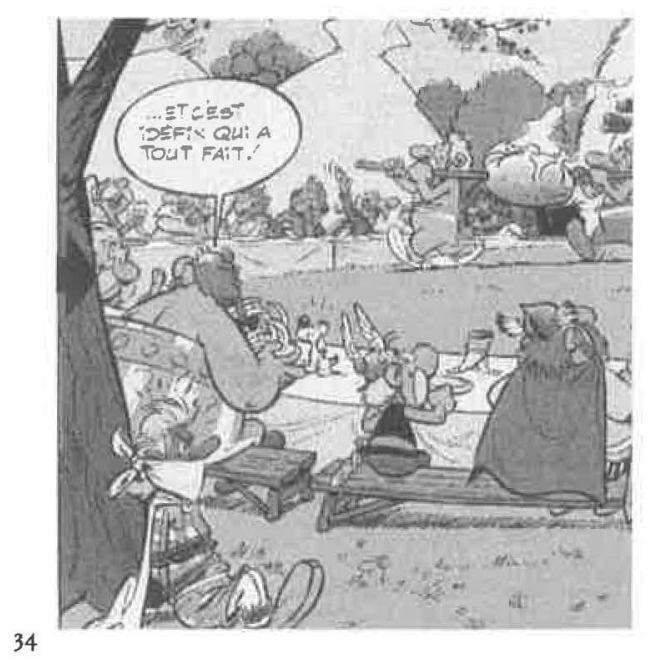

33. Asterix et les Normands, Paris, Hachette, 1999 (Dargaud, 1966), p. 43, c. 9.

34. Goscinny et Uderzo, Astérix et Cléopatre, Paris, Hachette, 1999 (Dargaud, 1965), p. 48, c. 5. (C) 2003 - Les Éditions Albert René / Goscinny-Uderzo. 
Contre la figure césarienne de l'auteur, maître légal des significations, Astérix questionne les postures d'interprètes du traducteur, de l'augure et de l'artiste porte-parole du pouvoir. Cependant la quête de l'identité auctoriale bascule vers des figures paradoxales d'enfant-auteur, œuvrant dans l'insu le plus total. L'exemple d'Obélix montre que rien n'empêche l'action la plus innocente de revêtir une portée collective quasi législatrice. Il semble alors qu'une double ambition auctoriale s'échafaude: il s'agit tout d'abord de ménager du jeu, en faisant mouche par un comique qui ne ferme pas le sens, mais aussi de créer une utopie narrative où se côtoient et s'affrontent différents systèmes institutionnels, en cernant au plus près ce qui les fait tenir. Face aux mythes d'auteur de la "grande" littérature, la seule légitimité sera celle de la culture comique et populaire de l'image. Un garant narratif permet cependant d'éviter le piège auctorial du narcissisme: le barde Assurancetourix, qui emblématise la catégorie de l'innommable au cœur même du langage. Avec ce personnage symbolique pour référence, gageons alors que la grande fête de la bande dessinée d'auteur peut enfin commencer. 